\title{
Hard wear-resistant coatings with anisotropic thermal conductivity for high thermal load applications
}

\author{
P. H. Michael Böttger, ${ }^{1,2, a)}$ Leonid Braginsky, ${ }^{1,3}$ Valery Shklover, ${ }^{1}$ Erik Lewin, ${ }^{2, b)}$ \\ Jörg Patscheider, ${ }^{2}$ David G. Cahill, ${ }^{4}$ and Matthias Sobiech ${ }^{5}$ \\ ${ }^{1}$ Laboratory of Crystallography, ETH Zürich, Vladimir-Prelog-Weg 5, 8093 Zürich, Switzerland \\ ${ }^{2}$ Laboratory of Nanoscale Materials Science, EMPA, Überland St. 129, 8600 Dübendorf, Switzerland \\ ${ }^{3}$ Institute of Semiconductor Physics \& Novosibirsk State University, 630090 Novosibirsk, Russia \\ ${ }^{4}$ Department of Materials Science and Engineering, University of Illinois, Urbana, Illinois 61801, USA \\ ${ }^{5}$ Oerlikon Balzers Coating AG, Iramali 18, 9496 Balzers, Principality of Liechtenstein
}

(Received 4 May 2014; accepted 19 June 2014; published online 3 July 2014)

\begin{abstract}
High thermal load applications such as high speed dry cutting lead to high temperatures in the coated tool substrate that can soften the tool and high temperature gradients that can put stress on the coating/tool interface. In this work, theoretical considerations are presented for multilayer and graded protective coatings that can induce a significant anisotropy in their thermal conductivity. Solution of the heat equation shows that anisotropy of thermal conductivity has the potential to reduce temperatures and temperature gradients arising due to brief, localized heat at the coating surface ("hot-spots"). Experimental realization of high anisotropy is demonstrated in TiN/AlCrN multilayer coatings with variable layer thickness. In the coating with $50 \mathrm{~nm}$ bilayer periodicity, the highest anisotropy was obtained with a value of $\kappa_{\|} / \kappa_{\perp}=3.0 \pm 0.9$. Time-domain thermoreflectance is used to measure in-plane and cross-plane thermal conductivity of fabricated coatings. The observed high values of anisotropy of thermal conductivity are compared with theoretical predictions and its realisation is discussed with regard to the coating microstructure. (C) 2014 AIP Publishing LLC.

[http://dx.doi.org/10.1063/1.4886182]
\end{abstract}

\section{INTRODUCTION}

Modern materials for high temperature, high mechanical loads, and high environmental demand applications such as Ti- and Ni-based superalloys are notoriously difficult to machine. This means high wear of machining tools and low material removal rate, both leading to high machining costs. One of the reasons for difficult machinability is the low thermal conductivity of these workpiece materials, creating high machining temperatures that result in softening and increased chemical reactivity of the tool, increased tool wear and even welding between tool and workpiece. ${ }^{1}$ The thermal issue is exacerbated by the desire for fast dry machining due to cost, environmental and health reasons, where the lack of cooling fluid leads to higher process temperatures. ${ }^{2}$

Design of thermal conductivity of tool coatings has only recently come into the focus of attention as a means to mitigate high temperature and thermal stresses at the tool surfaces during cutting. ${ }^{3,4}$ The rubbing contacts induce high heat loads in very small spots, whose size and distribution are dependent on pressure, cutting speed, the topography of the contact surfaces but also on the thermal properties of the tool/workpiece contact couple., ${ }^{5,6}$ Even though the thickness of a standard tool coating is only in the order of $5 \mu \mathrm{m}$, thermal design has been shown to improve performance, as higher amounts of heat can be deflected into the machining chip. ${ }^{7-10}$ Similarly, it has been shown that tribolayers forming during cutting can

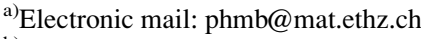

b) Present address: Department of Chemistry, Angström Laboratory, Uppsala University, Sweden.
}

have very low thermal conductance, thereby reducing heat flux into the tool. ${ }^{11}$ Consequently, changing the temperature distribution at the tool surface has the potential to decrease wear in high thermal load cutting applications. ${ }^{12}$ It has been shown analytically that in the idealized case of a point heat source at the coating surface, the anisotropy of thermal conductivity in the coating (where in-plane thermal conductivity is much higher than cross-plane thermal conductivity) can reduce in-plane thermal gradients at the tool surface significantly. ${ }^{13}$ In general, it can be expected that thermal design of the coating will be most effective for processes that involve short contact times and small areas such as milling and hobbing (gear cutting).

Many materials show appreciable values of anisotropy of thermal conductivity. But as we are interested in hard materials for coating applications, sapphire and silicon carbide with an intrinsic anisotropy value of only $\kappa_{\|} / \kappa_{\perp} \approx 1.1$ (Refs. 14 and $15)$ come to mind. Most of the widely used hard coatings consist of transition metal nitrides such as TiN and $\mathrm{CrN}$ and derivatives. These compounds crystallize in the cubic rocksalt-type structure $\mathrm{Fm} \overline{3} \mathrm{~m}$ (B1) that does not, by means of symmetry, result in anisotropic transport properties. Still, if materials are intrinsically isotropic, engineering them at the micro- and nanoscale allows to induce anisotropy by means of oriented crystallites, defects, grain boundaries or nanostructures ${ }^{16-18}$ or in multiphase systems, ${ }^{19}$ for example, oriented fibers embedded in a matrix. ${ }^{20}$ Out of these possibilities, for reasons of direct applicability, when using established industrial deposition processes for hard coatings, creating layered and graded coatings of intrinsically isotropic materials with different thermal conductivity to induce anisotropy is the focus of this work. 
The paper presents in Sec. II the theory of inducing anisotropy in coatings, using current technology and materials. Numerical evaluations of the heat equation are carried out to show the effect of coating anisotropy on temperatures and thermal gradients. In Sec. III, realization of an anisotropic system is demonstrated for a model multilayer TiN/AlCrN system where thermal conductivity and thermal anisotropy are measured directly. Comparing the results with model predictions provides an outlook on coatings with anisotropic thermal conductivity for cutting difficult-to-cut materials.

\section{THEORY}

\section{A. Induced anisotropy in multilayer and graded structures}

In order to lower peak temperatures during machining, heat should be dispersed parallel to the coating surface, while the heat flow perpendicular to it, i.e., towards the substrate should be minimized. Such a concept can be realized by multilayers consisting of single layers with different thermal conductivities. A layered coating can be seen as a stack of layers with thermal conductivity $\kappa_{i}$ and volume fraction $f_{i}$ with thermal interface resistances between individual layers $\rho_{i, i+1}$. The resulting total thermal conductivity parallel to the stack is given by

$$
\kappa_{\|}=f_{1} \kappa_{1}+f_{2} \kappa_{2}+f_{3} \kappa_{3}+\ldots,
$$

and thermal conductivity perpendicular to the layers by

$$
\frac{1}{\kappa_{\perp}}=\frac{f_{1}}{\kappa_{1}}+\rho_{1,2}+\frac{f_{2}}{\kappa_{2}}+\rho_{2,3}+\frac{f_{3}}{\kappa_{3}}+\ldots
$$

In the case of negligible interface resistance, a combination of two layers with $f_{1}=f_{2}=0.5$ (or periodic repetitions of this bilayer) results in an overall anisotropy $F$ of

$$
F \equiv \frac{\kappa_{\|}}{\kappa_{\perp}}=\frac{\left(\frac{\kappa_{1}}{\kappa_{2}}+1\right)^{2}}{4 \frac{\kappa_{1}}{\kappa_{2}}},
$$

where the in-plane conductivity is always higher than the crossplane conductivity as long as $\kappa_{1} \neq \kappa_{2}$. The interface resistance between the layers is neglected as, in the course of this study, only combinations of chemically similar transition metal nitrides are considered that are deposited in one continuous process. This should lead to very low interface resistance. ${ }^{21,22}$ As we aim for an increased anisotropy with $\kappa_{\|}>\kappa_{\perp}$, our negligence of the interface only leads to an underestimate of thermal anisotropy.
In contrast, combining different material classes such as metals with ceramics or amorphous with crystalline material would result in high interface resistance $\rho_{i, j}$ and by Eq. (2) to high thermal anisotropy. Engineering a practical hard coating would be difficult, however, due to the fundamental differences in thermal expansion, hardness and toughness and cannot be expected to yield coatings that are appropriate for high thermal and high mechanical load applications. The effects of finite thermal resistance and the possibility of manufacturing effective layered structures using industrial scale deposition techniques have been treated by the authors in a separate work. ${ }^{23}$

If the stack is replaced by a graded coating, the thermal conductivity becomes a one-dimensional function $\kappa(x)$ of distance or depth, defined between the top and bottom of the coating $x=0 \ldots d$. In the idealized case of a smooth and continuous function $\kappa(x)$, no interface and thus no interface resistance exists. The sums in Eqs. (1) and (2) can then be transformed into integrals

$$
\kappa_{\|}=\frac{1}{d} \int_{0}^{d} \kappa(x) d x \quad \text { and } \quad \frac{1}{\kappa_{\perp}}=\frac{1}{d} \int_{0}^{d} \frac{1}{\kappa(x)} d x .
$$

This results in the following functional for anisotropy in the graded material:

$$
F[\kappa(x)]=\frac{\kappa_{\|}}{\kappa_{\perp}}=\frac{1}{d^{2}} \int_{0}^{d} \kappa(x) d x \int_{0}^{d} \frac{1}{\kappa(x)} d x,
$$

which has analytical solutions for many forms of the thermal conductivity function $\kappa(x)$ as shown in Table I. Using the step function, that is equivalent to a multilayer, again results in Eq. (3). In the chosen dimensionless description of $k(x)$ in Table I, the resulting anisotropy depends only on the ratio $\alpha=\kappa_{\max } / \kappa_{\min }$ between the maximum and minimum thermal conductivity in the coating.

Such gradients, as listed in Table I, could be realized by depositing one of the following systems with designed oxygen gradients: $\mathrm{TiO}_{x} \mathrm{~N}_{1-x}(\alpha=7), \mathrm{CrO}_{x} \mathrm{~N}_{1-x}(\alpha=4.5),{ }^{24}$ and $\mathrm{AlO}_{x} \mathrm{~N}_{1-x}(\alpha \approx 15){ }^{25}$ These systems allow adjusting thermal conductivity via variation of oxygen concentration. Unfortunately, AIN is not suitable for many applications due to low hardness and rapid decomposition in air at elevated temperatures.

Combining different cubic transition metal nitrides, which can be assumed to form stable multilayer structures, ratios of up to $\alpha \approx 10$ should be attainable using TiN and AlCrN. ${ }^{26}$ Using Eq. (3), this multilayer structure would then feature an anisotropy of $F \approx 3$.

TABLE I. Analytical solutions for thermal anisotropy of coatings with gradient of thermal conductivity. For a dimensionless representation of $\kappa(x)$, the following definitions are used: $\alpha \equiv \kappa_{\max } / \kappa_{\min }, d \equiv 1, n \in \mathbb{Z}$.

\begin{tabular}{lcr}
\hline \hline Gradient type & $\kappa(x)$ & $\kappa_{\|} / \kappa_{\perp}$ \\
\hline Linear & $1+(\alpha-1) x$ & $\ln \alpha \cdot(\alpha+1) /(2 \alpha-2)$ \\
Quadratic & $1+(\alpha-1) x^{2}$ & $\arctan (\sqrt{\alpha-1}) \cdot(\alpha+2) /(3 \sqrt{\alpha-1})$ \\
Sinusoidal & $1+(\alpha-1)[\cos (2 n \pi x) / 2+1 / 2]$ & $(\alpha+1) /(2 \sqrt{\alpha})$ \\
Multilayer/Steps & $1+(\alpha-1) \Theta(x-1 / 2)$ & $(\alpha+1)^{2} /(4 \alpha)$ \\
\hline \hline
\end{tabular}


Calculated anisotropy as function of ratio $\alpha$ using the results presented in Table I is plotted in Figure 1, together with estimated ranges of $\alpha$ for real material systems (grey). The difference between the "Multilayer" and the "Sinusoidal" case in Figure 1 visualizes the negative consequences, which diffuse interfaces would have in multilayer materials, resulting in a significant reduction of the engineered anisotropy.

\section{B. Heat equation for anisotropic media}

Wear processes can be considered as caused by action of localized brief heat sources acting upon the coating/substrate system. ${ }^{27}$ In this picture, analytical tools can be used to evaluate the influence that coatings with a thermal anisotropy could have on temperature distribution in a wear-related process such as a sliding heat source. Analytical solutions for the isotropic case have been described. ${ }^{28,29}$ The temperature evolution in an anisotropic medium (the coating) due to an external heat source (for example, a friction contact) in cylindrical symmetry follows the heat equation

$$
\frac{\partial T}{\partial t}-\lambda_{\|} \frac{1}{r} \frac{\partial}{\partial r}\left(r \frac{\partial T}{\partial r}\right)-\lambda_{\perp} \frac{\partial^{2} T}{\partial z^{2}}=Q(r, z, t),
$$

where $T$ is temperature, $t$ is time, $r$ is the centrosymmetric radial coordinate, $z$ is the height coordinate, $\lambda_{\|}$is thermal diffusivity parallel to $r, \lambda_{\perp}$ is thermal diffusivity parallel to $z$, and $Q$ is the heat source. Thermal diffusivity is connected to thermal conductivity by $\lambda=\kappa / \rho c$ with $\rho c$ being the volumetric heat capacity. Volumetric heat capacity is assumed constant for the closely related transition metal (oxy)nitride materials considered in this work.

Application of this equation to the case of a point heat source (heat source described by Dirac $\delta$ function of $r, z$, and $t$ ) leads to an analytical solution that predicts significant reduction in thermal gradients parallel to the surface at the coating-substrate interface if $\kappa_{\|} / \kappa_{\perp}>1 .{ }^{13}$

The applied description of the heat source as point-like in space and time can be reasoned by considering the fact that in cutting applications rough hard surfaces are moving against each other, which creates a constantly changing

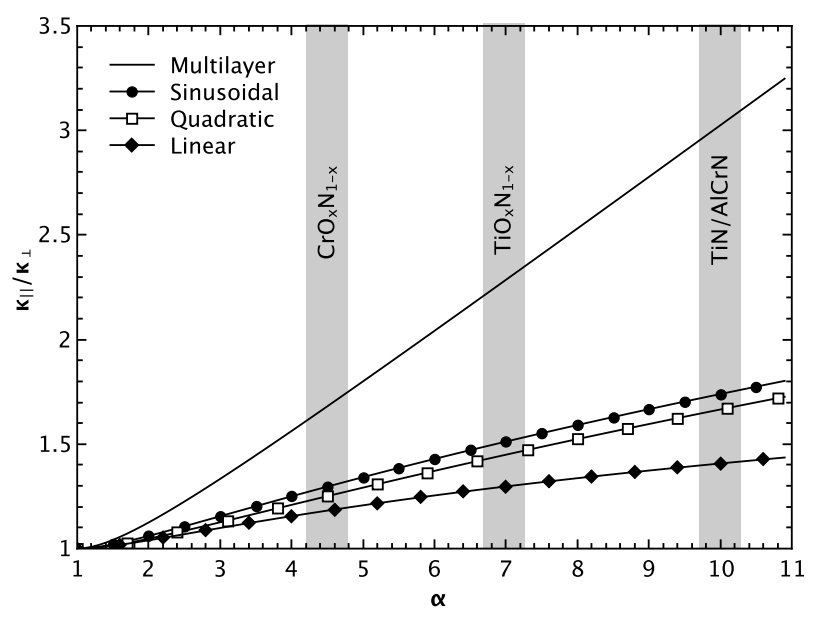

FIG. 1. Thermal anisotropy of coatings as a function of ratio $\alpha=\kappa_{\max } / \kappa_{\min }$ for different gradient functions listed in Table I with estimated range of $\alpha$ for selected material systems. topology, where only small asperities are briefly in real contact. $^{6,27}$

Still, the size of a contact may depend strongly on the type of process that the coating is subjected to (process pressure, workpiece roughness, coating hardness). It is then of interest to evaluate whether larger contacts can still be beneficially affected by an anisotropic coating. In order to model the effect of the heat created by a brief friction contact with a finite diameter, we here assume an instantaneous radially symmetric heat source with a diameter $2 R$ that is confined to the top of the coating. This can be described by using

$$
Q(r, z, t)=Q_{0} \Theta(r-R) \delta(z) \delta(t),
$$

using the Heaviside $\Theta$ function and the Dirac $\delta$ function with $Q_{0}$ as a scalar. Solving Eq. (6) in the half-space results in the following expression for the temperature increase:

$$
\begin{aligned}
\Delta T(r, z, t)= & \frac{Q_{0} \sqrt{\pi}}{4 \lambda_{\|} \sqrt{\lambda_{\perp}} t^{3 / 2}} \exp \left(-\frac{z^{2}}{4 \lambda_{\perp} t}\right) \\
& \times \int_{0}^{R} \exp \left(-\frac{r^{2}+r_{1}^{2}}{4 \lambda_{\|} t}\right) I_{0}\left(\frac{r r_{1}}{2 \lambda_{\|} t}\right) r_{1} d r_{1},
\end{aligned}
$$

where $I_{0}$ denotes the zero-order modified Bessel function. This equation can be evaluated numerically for different scenarios and its derivatives can be used to calculate temperature gradients as a function of heat source diameter $2 R$ over coating thickness $z$. This allows to calculate limitations of an anisotropic coating as the heat source becomes much wider than the coating thickness.

The change of the temperature distribution with thermal anisotropy $F=\frac{\kappa_{\|}}{\kappa_{\perp}}$ of the coating and heat source diameter $2 R$ is illustrated in Figure 2. A point-like heat source leads to spherical symmetry of heat distribution in an isotropic medium, as intuitively expected (Fig. 2(a)). This spherical symmetry becomes elliptic in shape for an anisotropic medium where in-plane heat transport is higher than cross-plane (Fig. 2(b)). The limitation of this beneficial spreading of heat becomes clear when an extended heat source is considered (Figs. 2(c) and 2(d)) where isotropic and anisotropic medium lead to similar shapes of the temperature distribution.

Using the solution for the heat equation (8), the benefit of an anisotropic coating can then be understood by calculating the reduction of the maximum temperatures and maximum temperature gradients at a certain depth in the medium. For our comparisons between isotropic and anisotropic coatings, we sensibly define a certain depth as the coating/substrate interface. Only the anisotropy of thermal conductivity and not the absolute value of its components becomes relevant here as we treat the substrate as a perfect heat sink. This is justified by the relatively high thermal conductivity of tool steels and carbides compared to transition metal nitride coatings.

Numerical results of the heat equation (8) are given in Figures 3 and 4 . They show that a model multilayer coating with high anisotropy (TiN/AlCrN multilayer, $F=3$ ) significantly reduces temperatures and gradients, even if the heat source is extended over more than the coating thickness. 

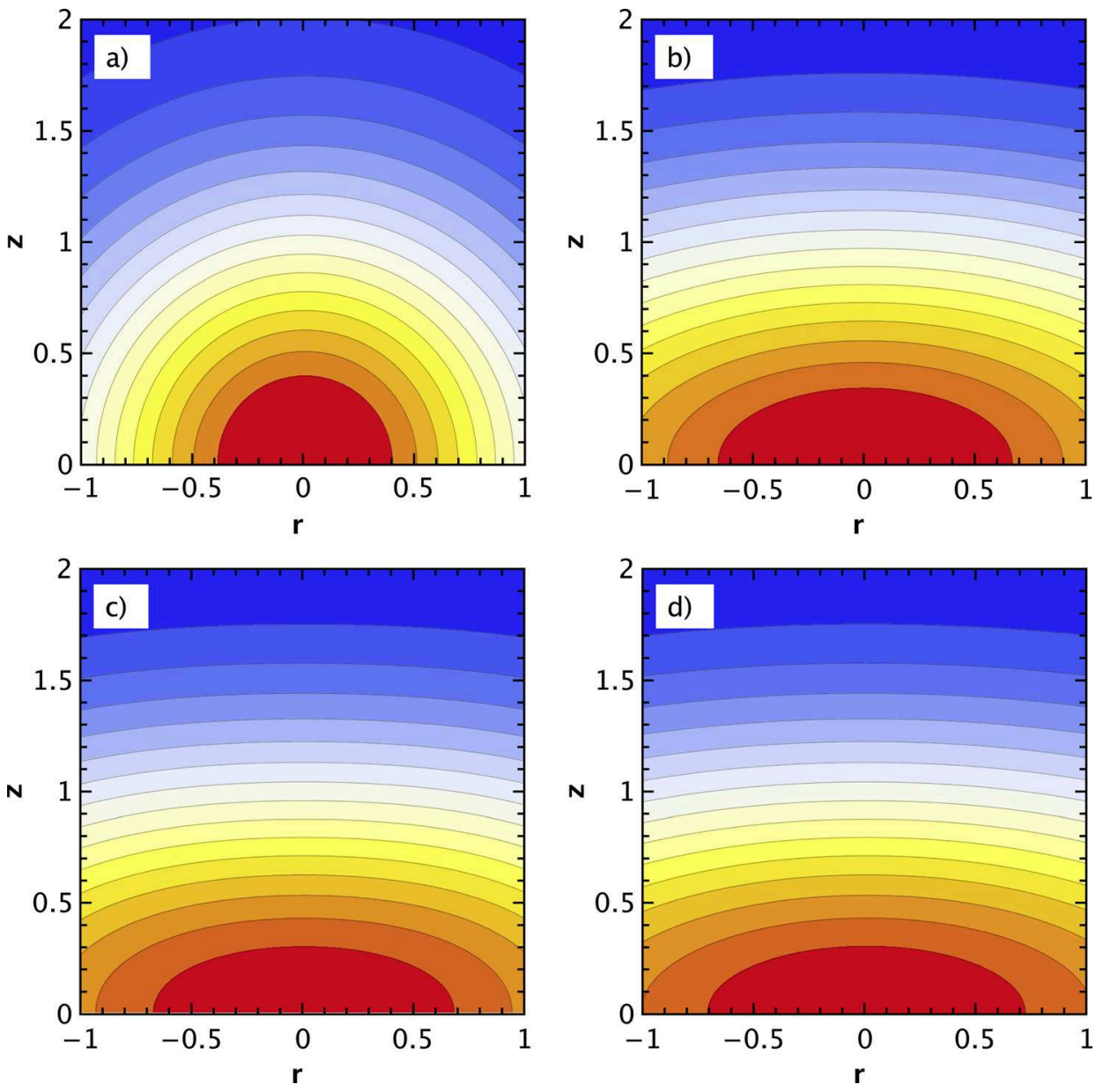

FIG. 2. Dimensionless visualization of temperature distribution using Eq. (8) with $Q_{0}=1, t=1$, and with the heat source centered at $r=z=0$. Unit of length for $r, z, R$ is 1 . Anisotropic $(F=3)$ and isotropic $(F=1)$ cases are compared in their response to small $(R=0.01)$ and large $(R=1)$ heat source diameter. Temperature is indicated by color from low (blue) to high (red). (a) Small heat source and isotropic medium, (b) small heat source and anisotropic medium, (c) large heat source and isotropic medium, and (d) large heat source and anisotropic medium. In the case of an extended heat source (c) and (d), thermal anisotropy does not significantly change the distribution.
This reduction is also seen in the model material with a sinusoidal gradient of thermal conductivity $\left(\mathrm{TiO}_{x} \mathrm{~N}_{1-x}, F=1.5\right)$. Evaluating any isotropic coatings with different values of thermal conductivity always results in the same maximum gradients and maximum temperatures, just at different points in time after introduction of the heat, depending on coating material thermal diffusivity.

The diminishing effect of an anisotropic coating on the temperature distribution with increasing heat source diameter can be understood as a transition to a 1D heat flow

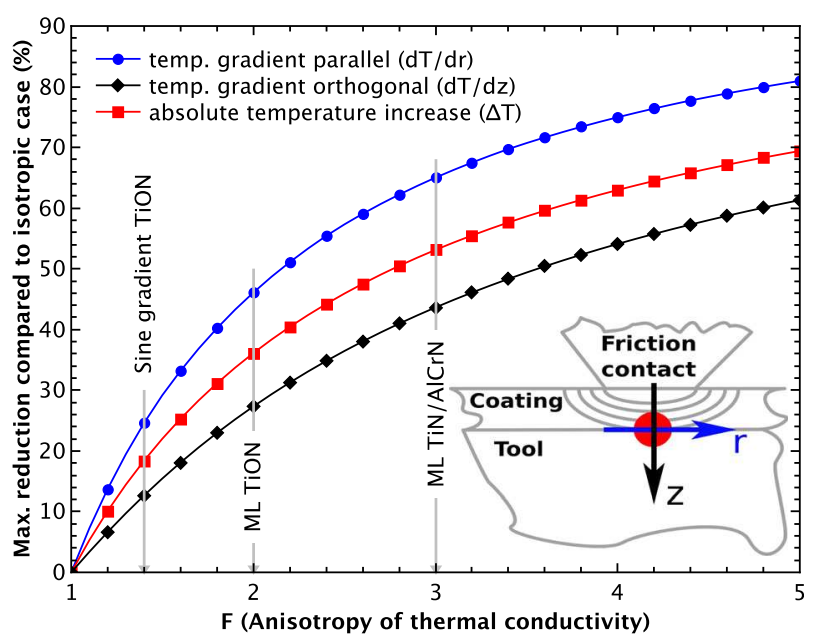

FIG. 3. Reduction of maximum temperature increase $\Delta T_{\max }$ and maximum temperature gradients at the coating/substrate interface parallel $(d T / d r)$ and perpendicular $(d T / d z)$ to the interface evaluated using Eq. (8) and its respective derivatives. Maximum reduction of the values resulting when choosing anisotropic coatings over any isotropic one as a function of anisotropy $F$ using $2 R / z=2$.

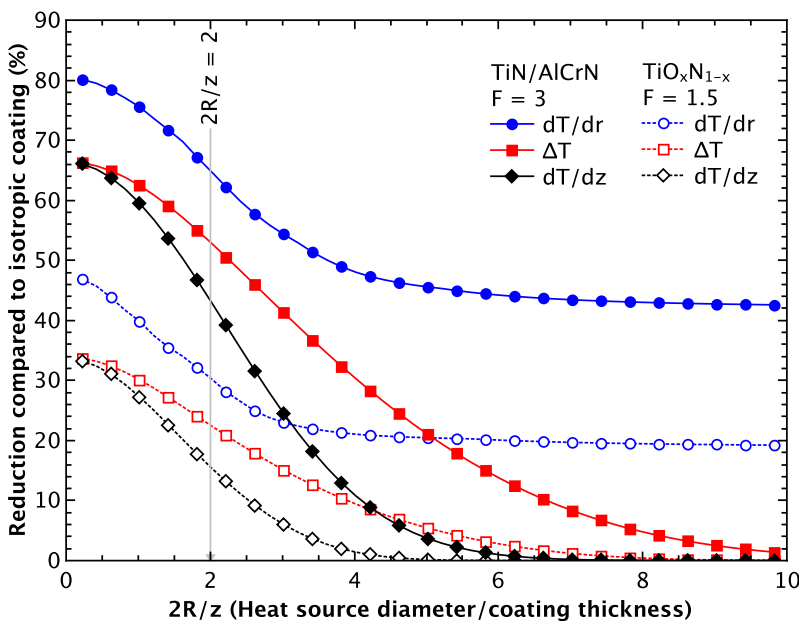

FIG. 4. Reduction of maximum temperature increase $\Delta T_{\max }$ and maximum temperature gradients at the coating/substrate interface parallel $(d T / d r)$ and perpendicular $(d T / d z)$ to the interface evaluated using Eq. (8) and its respective derivatives. Maximum reduction of the values resulting when choosing anisotropic coatings over any isotropic one as function of ratio between heat source diameter and coating thickness $2 R / z$ for example materials with $F=1.5$ and $F=3$. 
(compare also Figure 2). However, maximum temperature gradients $d T / d r$ along the radial coordinate are always significantly reduced, even for very wide heat sources. This is due to the finite size of the heat source. At its borders, where the highest radial gradients occur, the gradients are always being affected similarly by enhanced in-plane thermal transport, regardless of absolute dimensions.

It is difficult to directly predict specific performance enhancements of a coating just from these theoretical results. Reduction of gradients and temperatures is in these calculations strongly dependent upon the size of a typical brief friction contact (size of the heat source) and thereby upon the type and parameters of the process in question. The calculations show that in general anisotropic coatings lead to lower thermal gradients in the coating/substrate system and thereby could lead to less thermal load at the interface. In a different application, functionally graded coatings with variable thermal conductivity have been shown to beneficially affect the thermal cracking behaviour in partially stabilized zirconia coatings. $^{30}$

A limitation of our approach is the focus on instantaneous heat sources $[Q(r, z, t) \propto \delta(t)]$. For cutting applications, it would also be of interest to consider heat sources that are of finite diameter, moving and have finite duration. If the velocity of the moving heat source is known, the temperature distribution can be evaluated as a superposition of the solutions from the approach presented here (Eq. (8)). This may increase or decrease temperature gradients at the coating/ substrate interface depending on velocity, contact size, duration, and coating thickness. For very low velocities, it is expected that the beneficial reduction of temperatures and gradients by anisotropic coatings becomes less pronounced, as the temperature distribution transitions to a quasistatic regime.

\section{EXPERIMENTAL}

Experimental realization of an anisotropic multilayer coating was demonstrated using coatings with a combination of TiN and AlCrN layers with variable but equal individual layer thickness. These materials were chosen for their wide usage in cutting applications and for their chemical and structural similarity, while featuring thermal conductivity values that differ by an order of magnitude (about $30 \mathrm{~W} \mathrm{~m}^{-1} \mathrm{~K}^{-1}$ for TiN and $3 \mathrm{~W} \mathrm{~m}^{-1} \mathrm{~K}^{-1}$ for $\mathrm{AlCrN}^{26}$ ). Applying Eq. (3) from the simple resistor model leads to an expected anisotropy of $F \approx 3$ for this multilayer structure. It is possible to measure values of anisotropy of thermal conductivity of coatings in this range as described below.

Individual layer thickness was varied to estimate the influence of interlayer thermal resistance and to find out, whether a low amount of thick layers that are cheaper to fabricate, can still result in anisotropic coatings. Intrinsic roughness of arc evaporation coatings sets a lower limit to individual layer thickness that we estimate at about $50 \mathrm{~nm}$ when aiming for well-defined parallel layers. This value is also close to where transition metal nitrides can be considered bulk material with respect to their thermal conductivity. ${ }^{31}$ Note that hardness increasing effects in multilayers usually only emerge at layer thickness $<10 \mathrm{~nm}^{32}$

\section{A. Coating deposition and characterization}

The nitride coatings were deposited by reactive cathodic arc evaporation using an Oerlikon Balzers Innova industrial deposition tool. The AlCrN layer material selected is a Balzers AlCrN standard coating, while the TiN layer was deposited as described in Ref. 24 with lowest possible oxygen content for highest thermal conductivity. Mirror-polished cemented carbide substrates were coated at a temperature of about $T_{S}=450{ }^{\circ} \mathrm{C}$; the total coating thickness was usually $5 \mu \mathrm{m}$, but about $20 \mu \mathrm{m}$ for the samples used for in-plane thermal conductivity measurements. For XRD measurements at high temperatures in air, polished high speed steel substrates (steel grade 1.3343) were selected for their higher thermal stability. It is tentatively assumed that the choice of substrate does not affect the microstructure or composition of the coatings. Multilayer structures were created by alternating between the $\mathrm{Ti}$ and $\mathrm{AlCr}$ targets in the deposition chamber.

Thermal conductivity and anisotropy of thermal conductivity were measured using time-domain thermoreflectance (TDTR). ${ }^{33}$ To prepare the samples for measurement, they had to be mirror-polished (SiC on paper, 4000 grit, $<1 \mu \mathrm{m}$ particle size) and coated with $80 \mathrm{~nm}$ thin Pt films. The change of reflectivity with temperature in Pt serves as a thermometer in the TDTR method. The pump laser beam has a frequency of $9.8 \mathrm{MHz}$ and about $15 \mathrm{~mW}$ of power focused to a variable radius between 2.6 and $10 \mu \mathrm{m}$. After a variable delay time in the order of picoseconds, a probing laser measures the change in reflectance. The thermal penetration depth is in the order of a few hundred nanometres, much smaller than the beam diameter. To find thermal conductivity, the change in reflectance over delay time of the probing beam can be fit to a layer model of the sample. ${ }^{33}$ The details of the used setup have been described elsewhere and the uncertainty of the method lies in the range of $20 \%$ (Ref. 34) for the absolute value of thermal conductivity. Cross-plane thermal conductivity was measured with normal incidence of the laser beam onto the coating. In-plane thermal conductivity was measured by preparing cross-sections of $20 \mu \mathrm{m}$ thick coatings embedded in polymer and carefully scanning the beam using a small focus $(2.6 \mu \mathrm{m})$ along the edge to identify the coating region. Combining the measurements to determine anisotropy of thermal conductivity leads to a uncertainty in anisotropy in the order of $30 \%$. This sets a lower limit to the anisotropy that can reliably be measured to $F \gtrsim 1.5$.

Cross section images of freshly cleaved samples were taken using an ORION helium ion microscope from Carl Zeiss (HeFIB). This microscope works by focusing a He ion beam onto the sample and detecting secondary electrons that are being excited. The small size of the excited volume leads to very high resolution and surface detail as compared to standard scanning electron microscopy. The beam current used was $0.5 \mathrm{pA}$ at $30 \mathrm{kV}$.

In situ high temperature X-ray diffraction (HT-XRD) was carried out in a Panalytical X-Pert Pro system in 


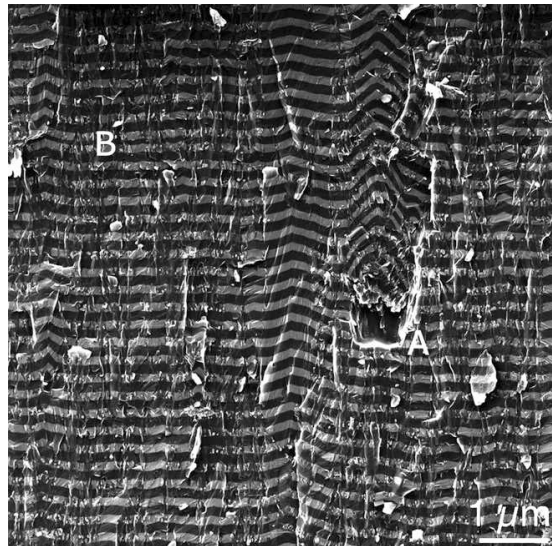

FIG. 5. Helium-ion microscopy image of cross section of $130 \mathrm{~nm} / 130 \mathrm{~nm}$ TiN/AlCrN. Annotated are common defect structures such as overgrown droplets (a) and inhomogeneous layer thickness (b).

Bragg-Brentano geometry and using a Ge(111) Johansson monochromator. An Anton Paar HTK 1200 environmental chamber was used to control temperature between $300 \mathrm{~K}$ and $1100 \mathrm{~K}$. All measurements were taken in air. Starting from room temperature, each diffraction pattern was taken during about $1 \mathrm{~h}$, after which the temperature was increased by $100 \mathrm{~K}$.

\section{B. Evaluation of multilayer microstructure}

Examination of cleaved cross sections was carried out in the HeFIB microscope in order to check the fabricated structures for defects and whether the planned microstructure could be realized (Fig. 5). The observed structure is close to the envisioned equally spaced parallel layers, but localized defects such as varying layer thickness and overgrown droplets do emerge. These may locally reduce the anisotropy of thermal conductivity of the coating. The layers are well defined, even in these rather thick coatings and feature very sharp interfaces, which are necessary for high anisotropy (see the discussion on gradients in Sec. II A). The cleaving process did not lead to separation of the layers, as the crack structures are continuously crossing many layers. This is in

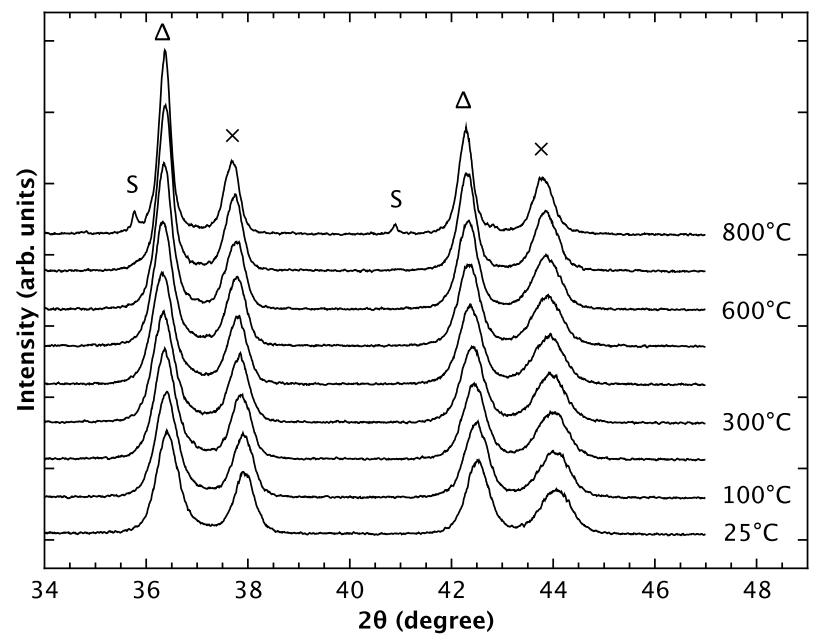

FIG. 6. Detail of high temperature in situ XRD measurements in air of a $130 \mathrm{~nm} / 130 \mathrm{~nm}$ TiN/AlCrN multilayer. $\Delta$ : TiN, $\times$ : AlCrN, S: Substrate (EN 1.3343 steel). contrast to specialized multilayer structures in hard coatings that feature beneficial crack arrest via deflection of crack growth along the layer direction. ${ }^{35}$

Employing XRD on the as-deposited coatings (Fig. 6, $25^{\circ} \mathrm{C}$ ) both components of the layer structure could separately be identified for $\mathrm{TiN}^{36}$ and with $\mathrm{AlCrN}$ peaks in between the diffraction peak locations of the alloy substituents cubic $\mathrm{AlN}^{37}$ and $\mathrm{CrN} .{ }^{38}$ Chemical integrity and stability of the multilayer structure were tested using high temperature XRD in air (Fig. 6). The cubic B1 phase of TiN and $\mathrm{AlCrN}$ could be clearly identified up to $800^{\circ} \mathrm{C}$, and no phase change or intermixing could be observed. It was not possible to measure at higher temperatures due to recrystallization and oxidation of the substrate (EN 1.3343 steel). As the top layer of the multilayer coating is $\mathrm{AlCrN}$, it effectively protects the TiN layers from oxidation that usually sets in at about $600{ }^{\circ} \mathrm{C} .^{39}$

\section{Measurement of thermal conductivity and thermal anisotropy}

TDTR is an established method for measuring crossplane thermal conductivity of hard coatings and multilayer structures, $3,40,41$ where the probing beam is incident normal to the coating surface. It is possible to measure anisotropy of thermal conductivity directly in such a measurement, but this requires very high quality samples and high values of anisotropy and has usually a considerable amount of uncertainty. ${ }^{42,43}$ For our samples of high roughness, low reflectance, and moderate values of anisotropy, we chose to measure in-plane thermal conductivity by scanning a focused beam at one fixed TDTR delay time over a cross section of the sample. The fixed delay time was chosen by sensitivity analysis ${ }^{41}$ to be insensitive to varying thermal conductance between reflective layer and sample and was, by calibration with normal incidence measurements, converted to thermal conductivity data.

A sample TDTR measurement, overlaid with a light microscopy image of the prepared cross section, is shown in Fig. 7. A local minimum of thermal conductivity appears inside the coating region (left side Fig. 7). This is a result

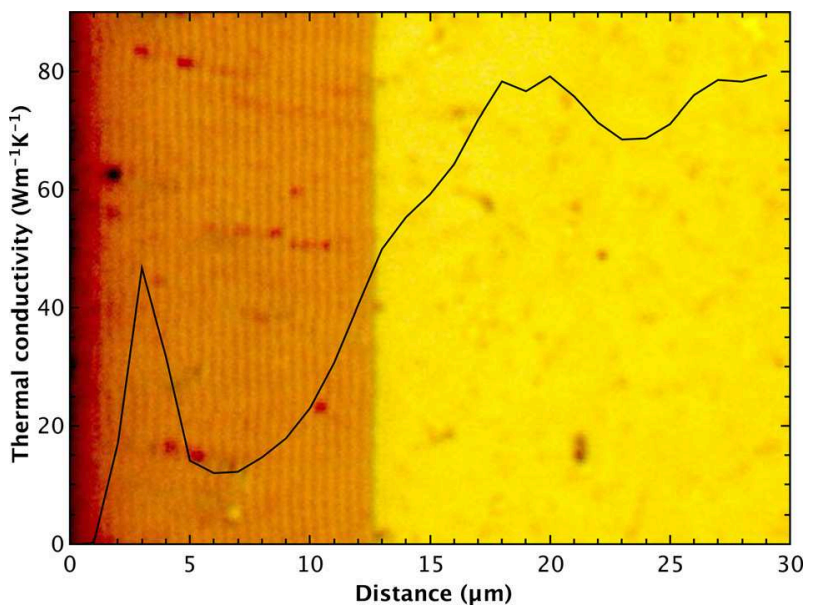

FIG. 7. Cross-section light microscopy image overlaid with result of scanning in-plane thermal conductivity of the $250 \mathrm{~nm} / 250 \mathrm{~nm}$ TiN/AlCrN sample using a $2.6 \mu \mathrm{m}$ beam width and $1 \mu \mathrm{m}$ step size. The two distinct brown and yellow areas visible are multilayer coating (left) and WC substrate (right). 
TABLE II. Measured values of thermal conductivity and anisotropy for multilayer samples. Monolayer reference sample is given for comparison.

\begin{tabular}{lccc}
\hline \hline Sample & $\kappa_{\|}\left(\mathrm{W} \mathrm{m}^{-1} \mathrm{~K}^{-1}\right)$ & $\kappa_{\perp}\left(\mathrm{W} \mathrm{m}^{-1} \mathrm{~K}^{-1}\right)$ & $F=\kappa_{\|} / \kappa_{\perp}$ \\
\hline $250 \mathrm{~nm}$ TiN/AlCrN & $8.5 \pm 1.7$ & $3.5 \pm 0.7$ & $2.4 \pm 0.7$ \\
$125 \mathrm{~nm}$ TiN/AlCrN & $11.7 \pm 2.3$ & $5.4 \pm 1.1$ & $2.2 \pm 0.7$ \\
$50 \mathrm{~nm}$ TiN/AlCrN & $12.2 \pm 2.5$ & $4.1 \pm 0.8$ & $3.0 \pm 0.9$ \\
Monolayer AlCrN & $2.9 \pm 0.6$ & $2.4 \pm 0.5$ & $1.2 \pm 0.4$ \\
\hline \hline
\end{tabular}

from the finite beam width and the very different thermal conductivities of embedding polymer to the left (not shown) and the substrate (right side of Fig. 7). We used the local minimum value of thermal conductivity in the coating region for evaluation. This is a source of uncertainty, but our following comparison with an isotropic coating serves as validation of this approach.

Measured values of thermal conductivity for multilayer TiN/AlCrN with varying bilayer thickness are listed in Table II and shown in Figure 8. The uncertainties are high, but it is clearly seen that anisotropy in TiN/AlCrN multilayer coatings is significant. The reference monolayer coating with no intrinsic anisotropy gave a measured value of anisotropy of $F=1.2 \pm 0.4$, compatible with the theoretical value $F=1$ to within experimental error (described in Sec. III A).

The anisotropy, that could be realized in these coatings, falls short of the value of $F \approx 3$ predicted by the resistor model for the coatings with 125 and $250 \mathrm{~nm}$ individual layer thickness. This could be due to microstructural defects exemplified in Fig. 5. Another possibility is diffusion at the interface between the individual layers that can reduce anisotropy (see Sec. II A). Of the coatings produced in this study, the one with $50 \mathrm{~nm}$ shows highest anisotropy. This may be due to interface resistance effects, but just as well due to the lower chance of incorporated macroscopic defects. Using Eqs. (1) and (2) and assuming equal interface conductance in all TiN/ $\mathrm{AlCrN}$ interfaces allows to extract its value from the measurements. Due to the high uncertainty of the measurements and the low number of data points, it was not possible to precisely determine the value of interface conductance, but it could be

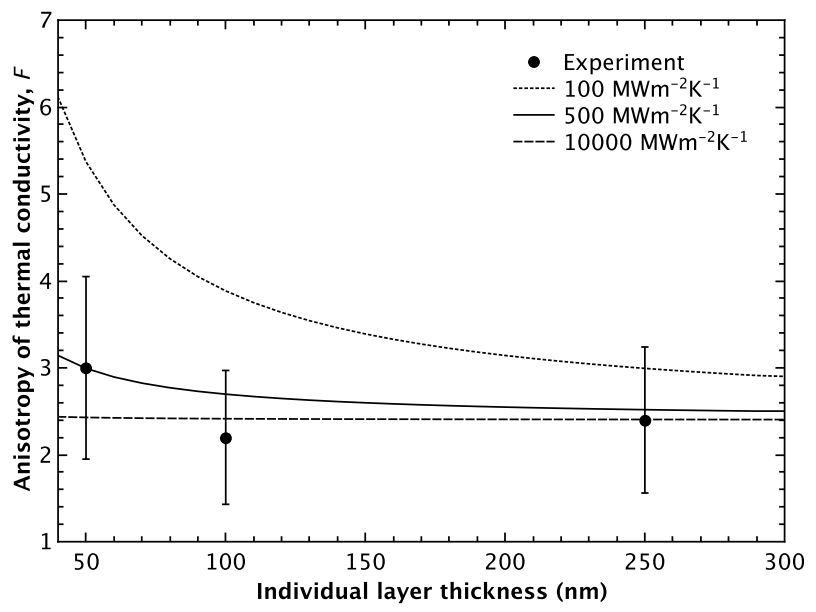

FIG. 8. Anisotropy of thermal conductivity in TiN/AlCrN multilayer samples (points) together with model results (lines) for varying values of interlayer thermal interface conductance. estimated to lie in the range of $300-2000 \mathrm{MW} \mathrm{m}^{-2} \mathrm{~K}^{-1}$ at room temperature, agreeing with reported measurements and estimates on combinations of materials of comparable chemical similarity. ${ }^{21,22}$ In this range, a modest $\approx 20 \%$ increase of anisotropy due to the interfaces could be engineered by choosing the lowest value for layer thickness, where roughness due to the arc-deposition method does not affect geometry significantly and cost of fabrication is manageable.

\section{CONCLUSION}

Hard coatings with highly anisotropic thermal conductivity are suggested based on combinations or variations of available coating materials. Experimental implementation of a model multilayer structure was done, and the achieved values of anisotropy are in line with theoretical predictions. These coatings are calculated to effectively spread the heat distribution inside the coating and thereby lower thermal gradients between coating and substrate. Future work will be necessary to translate this beneficial heat spreading mechanism to increased performance of engineered coatings in high thermal load applications.

\section{ACKNOWLEDGMENTS}

The authors would like to thank the SNSF R'equip (206021_133823) for the possibility to use the new He-Ion microscope at EMPA and Fabio La Mattina for operating it. Funding by the Competence Centre for Materials Science and Technology (CCMX) of the ETH Board (Switzerland) is gratefully acknowledged.

${ }^{1}$ E. O. Ezugwu, Int. J. Mach. Tools Manuf. 45, 1353 (2005).

${ }^{2}$ D. Dudzinski, A. Devillez, A. Moufki, D. Larrouqure, V. Zerrouki, and J. Vigneau, Int. J. Mach. Tools Manuf. 44, 439 (2004).

${ }^{3}$ W. Kalss, A. Reiter, V. Derflinger, C. Gey, and J. Endrino, Int. J. Refract. Met. Hard Mater. 24, 399 (2006).

${ }^{4}$ M. Nouari, G. List, F. Girot, and D. Coupard, Wear 255, 1359 (2003).

${ }^{5}$ P. Bogdanovich and D. Tkachuk, Tribol. Int. 39, 1355 (2006).

${ }^{6}$ D. Guha and S. K. Roy Chowdhuri, Wear 197, 63 (1996).

${ }^{7}$ W. Knig, R. Fritsch, and D. Kammermeier, CIRP Ann. 41, 49 (1992).

${ }^{8}$ W. Grzesik and P. Nieslony, J. Manuf. Processes 2, 79 (2000).

${ }^{9}$ J. Rech, A. Kusiak, and J. Battaglia, Surf. Coatings Technol. 186, 364 (2004).

${ }^{10} \mathrm{~W}$. Grzesik, in Advanced Machining Processes of Metallic Materials (Elsevier, Amsterdam, 2008) pp. 127-140.

${ }^{11}$ G. S. Fox-Rabinovich, J. L. Endrino, M. H. Aguirre, B. D. Beake, S. C. Veldhuis, A. I. Kovalev, I. S. Gershman, K. Yamamoto, Y. Losset, D. L. Wainstein, and A. Rashkovskiy, J. Appl. Phys. 111, 064306 (2012).

${ }^{12}$ H. Abdel-Aal, M. Nouari, and M. E. Mansori, Tribol. Int. 42, 359 (2009).

${ }^{13}$ L. Braginsky, A. Gusarov, and V. Shklover, Surf. Coat. Technol. 204, 629 (2009).

${ }^{14}$ C. Clauser and E. Huenges, Thermal Conductivity of Rocks and Minerals (American Geophysical Union, 1995).

${ }^{15}$ G.-P. Su, X.-H. Zheng, L. Qiu, D.-W. Tang, and J. Zhu, Int. J. Thermophys. 1, 1 (2012).

${ }^{16}$ J. Amrit, J. Phys. D: Appl. Phys. 39, 4472 (2006).

${ }^{17}$ J. E. Graebner, S. Jin, G. W. Kammlott, J. A. Herb, and C. F. Gardinier, Nature 359, 401 (1992).

${ }^{18}$ M. I. Flik, B. I. Choi, and K. E. Goodson, J. Heat Transfer 114, 666 (1992).

${ }^{19}$ S. Torquato, Random Heterogeneous Materials (Springer, 2001).

${ }^{20}$ J. Martinez-Garcia, L. Braginsky, V. Shklover, and J. W. Lawson, Phys. Rev. B 84, 054208 (2011).

${ }^{21}$ D. G. Cahill, A. Bullen, and S.-M. Lee, High Temp. - High Pressures 32, 135 (2000).

${ }^{22}$ H.-K. Lyeo and D. G. Cahill, Phys. Rev. B 73, 144301 (2006). 
${ }^{23}$ P. H. M. Böttger, A. V. Gusarov, V. Shklover, J. Patscheider, and M. Sobiech, Int. J. Therm. Sci. 77, 75 (2014).

${ }^{24}$ P. H. M. Böttger, E. Lewin, J. Patscheider, V. Shklover, D. G. Cahill, R. Ghisleni, and M. Sobiech, Thin Solid Films 549, 232 (2013).

${ }^{25}$ G. A. Slack, R. A. Tanzilli, R. O. Pohl, and J. W. Vandersande, J. Phys. Chem. Solids 48, 641 (1987).

${ }^{26}$ J. Martan and P. Benes, Thermochim. Acta 539, 51 (2012).

${ }^{27}$ K. Varadi, Y. Neder, K. Friedrich, and J. Flöck, Tribol. Int. 33, 789 (2000).

${ }^{28}$ B. Gecim, Wear 123, 59 (1988).

${ }^{29}$ Y. B. Hou and R. Komanduri, Int. J. Heat Mass Transfer 43, 1679 (2000).

${ }^{30}$ A. Kawasaki and R. Watanabe, Eng. Fract. Mech. 69, 1713 (2002).

${ }^{31}$ V. Rawat, Y. K. Koh, D. G. Cahill, and T. D. Sands, J. Appl. Phys. 105, 024909 (2009).

${ }^{32}$ M. Shinn, L. Hultman, and S. A. Barnett, J. Mater. Res. 7, 901 (1992).

${ }^{33}$ D. G. Cahill, Rev. Sci. Instrum. 75, 5119 (2004).
${ }^{34}$ K. Kang, Y. K. Koh, C. Chiritescu, X. Zheng, and D. G. Cahill, Rev. Sci. Instrum. 79, 114901 (2008).

${ }^{35}$ A. A. Voevodin, S. D. Walck, and J. S. Zabinski, Wear 203-204, 516 (1997).

${ }^{36}$ A. N. Christensen, Acta Chem. Scand. A 32, 89 (1978).

${ }^{37}$ N. E. Christensen and I. Gorczyca, Phys. Rev. B 47, 4307 (1993).

${ }^{38}$ M. N. Eddine, E. F. Bertaut, M. Roubin, and J. Paris, Acta Crystallogr., Sect. B 33, 3010 (1977)

${ }^{39}$ W.-D. Münz, J. Vac. Sci. Technol. A 4, 2717 (1986).

${ }^{40}$ G. S. Fox-Rabinovich, K. Yamamoto, M. H. Aguirre, D. G. Cahill, S. C. Veldhuis, A. Biksa, G. Dosbaeva, and L. S. Shuster, Surf. Coatings Technol. 204, 2465 (2010).

${ }^{41}$ D. G. Cahill, W. K. Ford, K. E. Goodson, G. D. Mahan, A. Majumdar, H. J. Maris, R. Merlin, and S. R. Phillpot, J. Appl. Phys. 93, 793 (2003).

${ }^{42}$ J. P. Feser and D. G. Cahill, Rev. Sci. Instrum. 83, 104901 (2012).

${ }^{43}$ A. J. Schmidt, X. Chen, and G. Chen, Rev. Sci. Instrum. 79, 114902 (2008). 